\title{
Inverse Compton Scattering in pulsar physics
}

\author{
G.J. Qiao \\ Department of Geophysics, Peking University, Beijing 100871, China
}

\begin{abstract}
Inverse Compton Scattering (ICS) is a very important process not only in inner gap physics, but also for radio emission. ICS of high energy particles with thermal photons is the dominant and a very efficient mechanism of the particle energy loss above the neutron star surface, and is an important process in causing gap breakdown. The pulsar distribution in the $P-P$ diagram and the observed mode changing phenomenon of some pulsars can be expained by the sparking conditions due to ICS. ICS of the secondary particles with the low frequency wave from the inner gap sparking can be responsible for radio emission. In this ICS model, many observational features of pulsar radio emission can be explained, such as: one core and two conal emission components, their different emission altitudes and relative time delay effects; spectral behavior of pulse profiles; the behavior of the linear polarization and position angle.
\end{abstract}

\section{ICS of thermal photons near neutron star surface}

In the strong magnetic field above the surface of a neutron star, it has been verified by many authors that the ICS process is the dominant mechanism for the generation of high energy photons, such as gamma-rays, and for energy loss of relativistic particles (Xia et al. 1985; Daugherty \& Harding 1989; Dermer 1990; Preece \& Harding 1992; Bednarek et al. 1992; Zhang \& Qiao 1995; Chang 1995; Sturner 1995).

Xia et al. (1985) find that (a) the total cross section of ICS in strong magnetic field is greatly increaced near the resonant freguency; (b) ICS has a higher efficiency than curvature radiation, so that it is an important mechanism for the energy loss of high energy particles, and in some cases it can limit the acceleration of the particles; (c) ICS may be the most important mechanism for producting high energy photons. Daugherty \& Harding (1989) show that if the magnetic field is not considered, electrons will lose substantial energy only when the temperature of the neutron star surface is very high $\left(T>3 \times 10^{6} \mathrm{~K}\right)$. If a strong magnetic field is present $\left(B \sim 10^{12} \mathrm{G}\right)$, resonant scattering will greatly increase electron energy-loss, making scattering very efficient even with a low surface temperature. Stuner(1995) finds that magnetic Compton scattering is the dominant energy-loss process when the electron Lorentz factors are less than a few times $10^{6}$. 


\subsection{Gap sparking conditions with the ICS process present}

In most pulsar radiation models, a neutron star turns off as a radio pulsar when it can no longer produce pairs (Chen \& Ruderman 1993). Taking the ICS process into account for inner gap sparking, Zhang \& Qiao (1995) found that in most cases ICS process will make the pair cascade occur earlier than the curvature radiation (CR) process, so that the basic properties of the gap, such as gap height or the potential drop across the gap, change greatly. The Lorentz factors of the out going particles should be smaller than previously thought, which makes the "binding energy problem" less problematic.

\subsection{Pulsar birth line, appearance line and death line}

Qiao \& Zhang $(1994,1996)$ investigate the pulsar distribution on $P-\dot{P}$ diagram with ICS process. With a dipole magnetic field, they get a theoretical death line, below which no pulsars have been observed. They also find two other lines: a birth line and an appearance line. The "birth line" is the line above which the gamma-photons produced in the gap have a perpendicular energy with respect the magnetic field of less than $2 m c^{2}$ so that the gap will not discharge. Neutron stars above the line can not be observed as radio pulsars. The "appearance line", delineates where the perpendicular magnetic field with respect to the photon direction is too small to cause magnetic pair production, so no neutron star appears as a pulsar under this line. The so-called Hubble-line is close to the appearance line.

\subsection{A possible explanation for mode changing phenomenon}

The mode changing phenomenon of some pulsars could be interpreted as the switching effect between the resonant ICS sparking mode and the thermal ICS sparking mode. These two mechanisms causing gap-breakdown can result in different Lorentz factors of the outflowing secondary particles as well as the different repeating frequencies of gap breakdown (Zhang et al 1996). Either or both of them would make the pulsar radio emission beam and emission regions changed.

\section{An ICS model for pulsar radio emission}

Qiao (1988a) estimated that the efficiency of the ICS process of low frequency waves with secondary particles is about $10^{14}$ times larger than the efficiency of curvature radiation for typical pulsar parameters. The efficiency can be estimated through several approachs. All calculations show that curvature radiation is a very inefficient mechanism compared with ICS process.

In the ICS model for pulsar radio emission, we have following assumptions: neutron stars have dipolar magnetic fields; low-frequency waves are generated from the repeated inner gap breakdown; the low-frequency wave can propagate through possible charge-separated plasma freely; the high energy particles moving along the magnetic field lines scatter the low frequency waves to produce the radio emission we observed. 


\subsection{Core and conal emission}

Core and conal emission components have been identified from observations (Rankin 1983, Lyne \& Manchester 1988). The radiation models based on curveture radiation can only give out one hollow cone. The same possible explanation of core emission has been proposed by Qiao $(1988,1992)$, Beskin et al. (1988), Wang et al. (1988) and Zhu et al (1994).

In the ICS model, with the above assumptions, we can get core and conal emission naturaly (Qiao 1992). Long period pulsars prefer to have an emission beam of one core and two cones, while fast pulsars prefer to have a beam of one core and only one cone. This agrees with observed profile morphology well (Rankin 1993, Gil \& Han 1996).

\subsection{Emission heights, retardation effects and polarization angle}

Rankin(1993) proposes that the "inner" cone is emitted at a lower height along the same group of dipole field lines that produce "outer" cone. It is true in ICS model (Qiao et al. 1992; Lin \& Qiao 1995).

If the core and conal emission are emitted at different heights, aberration and retardation effects should move the appearent position of the core beam to later longitude with respect to that of cone. Two effects can be seen: one is the shape of the pulsar emission beam (Lin \& Qiao 1995), and one is the linear polarization behaviour. The simulations of Xu et al. (1996) are helpful in understanding the variation of position angle in integral profiles, such as position angle jumps.

\subsection{Pulse profiles changing with frequency}

The frequency dependence of pulse profiles may bring us important information about the emission mechanism and geometry.

With the curvature radiation process and polar cap model, the frquency behaviour of pulse profiles of some pulsars such as PSR B0525+21 (conal double profile) can be explained, but that of much more other pulsars cannot. For example, the pulse profiles of PRS B0950+08 have two components at low frequencies, but only one at high frequencies (cf. Phillips \& Wolszczan 1992). In contrast, PSR B1642-03 has three components at high frequencies but one at low frequencies (Kramer 1994; Lyne \& Manchester 1988). Five-component profiles cannot be explained by the CR model anyway. However, all of these features can be explained by the ICS model (Qiao 1992). The frequncy dependence of pulse profiles can be read from the frequency-beam angle diagram (Qiao 1992). Multi-frequency observational data analysis, independently done by Wu et al. (1992), shows such a dependence.

\section{Conçlusions}

ICS process has been proved by many authors as being the most important mechanism for gamma-ray production and for the energy loss of the particles above the neutron star surface. Comparing with ICS, the curvature radiation is a very ineffective emission mechanism. Any theory for pulsar emission from nearby polar cap regions should take the influence of the ICS process into account. 
It has been very clear that because of the presentation of ICS process, the structure and parameters of the inner gap are quite different to those of earlier work, which is perhaps related to many observational facts.

The ICS process of low frequency wave coming from inner gap sparking with high energy out-flowing particles could be responsible for radio emission of pulsars, and many observational facts of pulsar radio emission could be explained in the ICS model.

Acknowledgments. I thank very much to Drs. Manchester R.N., Melrose D., Han J.L., Usov V., Asseo E., Machabeili G., Luo Q.H., Eilek J., and Malofeev V. for their valuable suggestions and discussions. I would also like to thank Mrs. Zhang B., Xu R.X., and Lin W.P. for many discussions. This work is supported partly by the NNSF of China, the Climbing Project, and the Doctoral Program Foundation of Higher Education in China.

\section{References}

Beskin V.S., Gurevich A.V., Istomin Y.N., 1988, ApSS 38, 437

Bednarek W., Cremonesi O., Treves A., 1992, ApJ 390, 489

Chang H.K., 1995, A\&A 301, 456

Daugherty J.K., Harding A.K., 1989, ApJ 336, 861

Dermer C.D., 1990, ApJ 360, 197

Gil J.A., Han J.L., 1996, ApJ 458, 265

Lin W.P., Qiao G.J., 1995, preprint

Kramer M., 1994, A\&A S 207, 525

Lyne A.G., Manchester R.N., 1988, MNRAS 234, 477

Phillips J.A., Wolszczan A., 1992, ApJ 385, 273

Preece R.D., Harding A.K., 1992, ApJ 386, 308

Qiao G.J., 1992, Proc. IAU Collo.128, p.238

Qiao G.J., 1988a, In: High Energy Astrophysics, Bonner G.B.(ed.) p.88

Qiao G.J., 1988b, Vistas in Astronomy 31, 393

Qiao G.J., Li C.G., Li M., 1992, Proc. IAU Collo.128, p243

Qiao G.J., Zhang B., 1994, Proc. 8th Guo Shoujing Summer School, in press

Qiao G.J., Zhang B., 1996, A\&A 306, L5

Rankin J.M., 1983, ApJ 274, 333

Rankin J.M., 1993, ApJ 405, 285

Sturner S.J., 1995, ApJ 446, 292

Wang D.Y., Wu X.J., Chen H., 1988, Vistas in Astronomy 31, 399

Wu X.J., Xu W., Rankin J.M., 1992, Proc. IAU Collo.128, p172

Xia, X.Y., Qiao G.J., Wu, X.J., and Hou Y.Q., 1985, A \& A, 152, 93

Xu R.X., Qiao G.J., Han J.L., 1996, Proc. IAU Collo.160, this volume.

Zhang B., Qiao G.J., 1995, A\&A, in press

Zhang B., Qiao G.J., Lin W.P., Han J.L., 1996, IAU Collo.160, this volume.

Zhu Y., Mao D., Wang D., Wu X., 1994, A\&A 282, 467 